\title{
Magnetic Resonance Morphometry of Normal Cerebral Aqueduct in South Indian Population
}

\author{
Veeramani Raveendranath ${ }^{1}$ Prafulla Kumar Dash ${ }^{2}$ Thangaraj Kavitha ${ }^{1} \quad$ Krishnan Nagarajan $^{3}$ \\ ${ }^{1}$ Department of Anatomy, Jawaharlal Institute of Postgraduate \\ Medical Education \& Research, Puducherry, India \\ ${ }^{2}$ Department of Radio-Diagnosis, Kalinga Institute of Medical \\ Sciences, Bhubaneswar, India \\ Address for correspondence Krishnan Nagarajan, MD, \\ DM, Department of Radio-Diagnosis, Jawaharlal Institute of \\ Postgraduate Medical Education \& Research, Dhanvantri Nagar, \\ Puducherry 605006, India (e-mail: Iknagarajan1@gmail.com).
}

${ }^{3}$ Department of Radio-Diagnosis, Jawaharlal Institute of

Postgraduate Medical Education \& Research, Puducherry, India

Indian J Neurosurg 2022;11:248-252

\begin{abstract}
Keywords

- cerebral aqueduct

- aqueduct of Sylvius

- morphometry

- magnetic resonance imaging

Introduction Cerebral aqueduct (of Sylvius) connects the third and fourth ventricles of the brain, and the shape of the aqueduct varies. The aim of the study is to assess the morphometry of the cerebral aqueduct in normal south Indian adult population and to look for pattern by shape, if any.

Materials and Methods One hundred and fifty normal brain MR images (75 males and 75 females) using 3D heavily T2-weighted sequence were analyzed for various normal parameters of cerebral aqueduct. Mean and standard deviation were calculated. Based on the shape of the aqueduct, an attempt was made to classify them. Unpaired t-test was used to assess any significant difference between age groups and gender. The intraclass coefficient correlation was used to analyze the interobserver variability. Results The mean value of the length of the cranial and caudal part of aqueduct in males were $0.69 \mathrm{~cm}$ and $0.86 \mathrm{~cm}$ and in females $0.65 \mathrm{~cm}$ and $0.80 \mathrm{~cm}$, respectively. The length was more in males, and it was statistically significant $(p=0.006$ and 0.02$)$. There are four types of cerebral aqueduct based on shape.

Conclusion MRI is considered as the investigation of choice for preoperative planning of brain operative procedures. The MR morphometric evaluation of cerebral aqueduct provides precise knowledge about the anatomy and may be of help in the diagnosis and treatment by endoscopic neurosurgery.
\end{abstract}

\section{Introduction}

Cerebral aqueduct (aqueduct of Sylvius) is a small tubular structure connecting the third and fourth ventricle for the passage of cerebrospinal fluid (CSF). ${ }^{1}$ Blockage of cerebral aqueduct or narrowing of aqueduct (aqueductal stenosis) leads to ventriculomegaly. ${ }^{2}$ Few studies have been conducted in postmortem specimens, cadavers, and plastic cast to determine the morphometry of cerebral aqueduct. Due to shrinkage of the ex vivo specimen, the dimensions obtained by those methods are considered equivocal. Nowadays, MRI is mostly considered as the investigation of choice for preoperative planning for brain operative procedure. So, MR morphometric evaluation of cerebral aqueduct may provide precise knowledge about anatomy and help to diagnose aqueductal stenosis. In the literature, there is a paucity of normal morphometric data in relation to cerebral aqueduct based on MRI. As the cerebral aqueduct descends from the third ventricle to fourth ventricle, the shape of the aqueduct

(C) 2022. Neurological Surgeons' Society of India.

This is an open access article published by Thieme under the terms of the Creative Commons Attribution-NonDerivative-NonCommercial-License, permitting copying and reproduction so long as the original work is given appropriate credit. Contents may not be used for commercial purposes, or adapted, remixed, transformed or built upon. (https://creativecommons.org/licenses/by-nc-nd/4.0/).

Thieme Medical and Scientific Publishers Pvt. Ltd. A-12, 2nd Floor, Sector 2, Noida-201301 UP, India 
varies. But it does not follow the same pattern. So, the aim of the study is to look for morphometry of aqueduct in normal adults and attempt to classify the cerebral aqueduct, according to the shape, among the south Indian population.

\section{Materials and Methods}

The study was retrospectively done on MRI images of brain, which were acquired in 1.5 Tesla MR equipment (Avanto Magnetom Siemens, Erlangen Germany) using 14-channel Head-coil. High-resolution heavily T2-weighted 3D sequence Sampling Perfection with Application optimized Contrasts using different flip-angle Evolution (SPACE) was used to acquire thin 1-mm sections. The scanning parameters were TR/TE-1200/162 msec; section thickness $1 \mathrm{~mm}$; no. of sections-120; FOV $220 \mathrm{~mm}$; flip angle 150*; matrix $192 \times 256$ with isotropic voxel size of $1 \times 1 \times 1 \mathrm{~mm}$ ). Thin section T1-weighted MPRAGE sequence was also acquired for comparison wherever necessary. The direct sagittal plane was used for better in-plane resolution. True sagittal sections were confirmed by the presence of stalk, infundibular recess, cerebral aqueduct and anterior commissure-posterior commissural line (AC-PC line) in the same visualized midsagittal plane image.

One hundred and fifty MR images (75 males and 75 females) that were reported to be normal were taken up for evaluation. The images were retrieved from picture archiving and communication system (PACS) after deidentification. The study was approved by the institute research and institute ethics committee.

MR images of age group between 18 and 60 years were studied. The images with any pathology involving the aqueduct, third and fourth ventricular region were excluded from the study. The parameters were measured thrice and the mean was taken as final. The same was measured also by a second observer to determine the interobserver variability.

In sagittal view, the shape of the cerebral aqueduct was noted and the following measurements were taken:

i. Length of the cerebral aqueduct: cranial part and caudal part separated by midangulation ${ }^{3}$

ii. Diameter of cerebral aqueduct: proximal end (D1), middle part (D2), distal end (D3) (-Fig. 1A) at upper, mid- and distal third of aqueduct iii. Angles of cerebral aqueduct: Angle between line along the floor of the third ventricle tangential to cranial opening of the aqueduct and the cranial part of aqueduct (A1); angle between cranial and caudal parts of aqueduct (A2); angle between the caudal part of aqueduct and the floor of the fourth ventricle (A3) ( - Fig. 1B). As the cranial opening is important in terms of endoscopic procedures and orientation of aqueduct to third ventricular floor may vary, tangential line was taken for A1 angle measurement.

Mean and standard deviation were calculated for all the measurements. Unpaired t-test was used to evaluate the statistical difference between the genders $(p<0.05)$. The intraclass coefficient correlation was used to evaluate the interobserver variability.

\section{Results}

The mean measurements of length, diameter at three levels and the angles of the aqueduct are presented in the - Table 1. The mean value of the length of the cranial and caudal part of aqueduct in males were $0.69 \mathrm{~cm}$ and $0.86 \mathrm{~cm}$ and in females $0.65 \mathrm{~cm}$ and $0.80 \mathrm{~cm}$, respectively. The difference in the lengths of the cranial and caudal parts between males and females (more in males) was statistically significant ( $p=0.006$ and 0.02 , respectively). The mean diameters of the aqueduct at cranial, middle and caudal parts in males were $0.12 \mathrm{~cm}, 0.13 \mathrm{~cm}$ and $0.18 \mathrm{~cm}$, respectively. The mean diameters of aqueduct at cranial, middle and caudal parts in females were $0.12 \mathrm{~cm}, 0.13 \mathrm{~cm}$ and $0.17 \mathrm{~cm}$, respectively.

There are four types of cerebral aqueduct based on the shape. Based on these findings, the aqueduct could be classified into following types ( - Fig. 2):

Type I: Diameter at all three levels (cranial, middle and caudal part) are same.

Type II: Diameters at cranial and middle parts are same which is less than the caudal part.

Type III: Diameter at cranial and caudal parts are same which is less than the middle part.

Type IV: Morphology other than type I, II, III.

The percentage of each type has been given in - Table 2 . The most common type of aqueduct in males was type I (60\%) followed by type II (25\%) and type III (8\%). But in females,

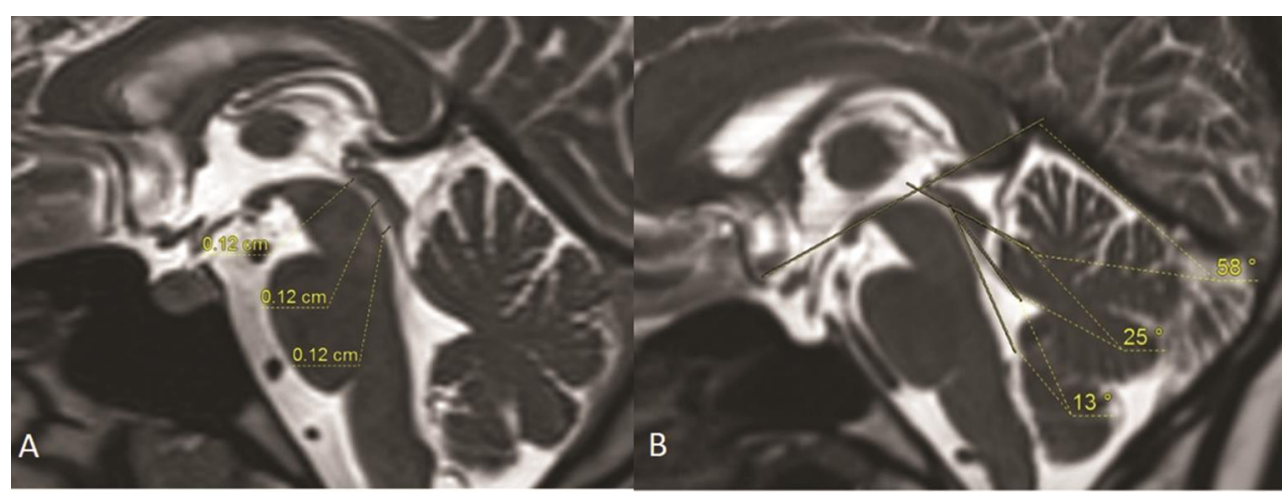

Fig. 1 T2-weighted 3D Sampling Perfection with Application optimized Contrasts using different flip-angle Evolution (SPACE) MRI images of brain in sagittal plane showing the measurement of the diameter of cerebral aqueduct at upper, mid and lower parts (A) and the various angles (B) mentioned in the methods. 
Table 1 Mean values of parameters of cerebral aqueduct (length and diameter in $\mathrm{mm}$, angle in degree)

\begin{tabular}{|c|c|c|c|c|c|c|c|}
\hline \multirow[t]{2}{*}{ S.no } & \multicolumn{2}{|c|}{ Parameters } & \multicolumn{2}{|l|}{ Males } & \multicolumn{2}{|l|}{ Females } & \multirow[t]{2}{*}{ Significance } \\
\hline & & & Mean \pm SD & Range & Mean \pm SD & Range & \\
\hline \multirow[t]{2}{*}{1} & \multirow{2}{*}{$\begin{array}{l}\text { Length of } \\
\text { aqueduct }\end{array}$} & Cranial part (L1) & $6.9 \pm 1.1$ & $6.7-7.2$ & $6.5 \pm 0.9$ & $6.3-6.7$ & 0.006 \\
\hline & & Caudal part (L2) & $8.6 \pm 1.4$ & $8.2-9.0$ & $8.0 \pm 1.1$ & $7.8-8.3$ & 0.02 \\
\hline \multirow[t]{3}{*}{2} & \multirow[t]{3}{*}{ Diameter } & Cranial end (D1) & $1.2 \pm 0.2$ & $1.2-1.3$ & $1.2 \pm 0.2$ & $1.2-1.3$ & 0.44 \\
\hline & & Middle part(D2) & $1.3 \pm 0.3$ & $1.2-1.4$ & $1.3 \pm 0.2$ & $1.2-1.4$ & 0.73 \\
\hline & & Caudal end (D3) & $1.8 \pm 0.5$ & $1.7-1.9$ & $1.7 \pm 0.5$ & $1.5-1.8$ & 0.06 \\
\hline \multirow[t]{3}{*}{3} & \multirow[t]{3}{*}{ Angle } & Angle 1 (A1) & $51 \pm 3$ & $42-59$ & $53 \pm 4$ & $52-54$ & 0.06 \\
\hline & & Angle 2 (A2) & $28 \pm 3$ & $20-36$ & $26 \pm 3$ & $25-27$ & 0.06 \\
\hline & & Angle 3 (A3) & $12 \pm 2$ & $11-13$ & $12 \pm 2$ & $11-13$ & 0.39 \\
\hline
\end{tabular}

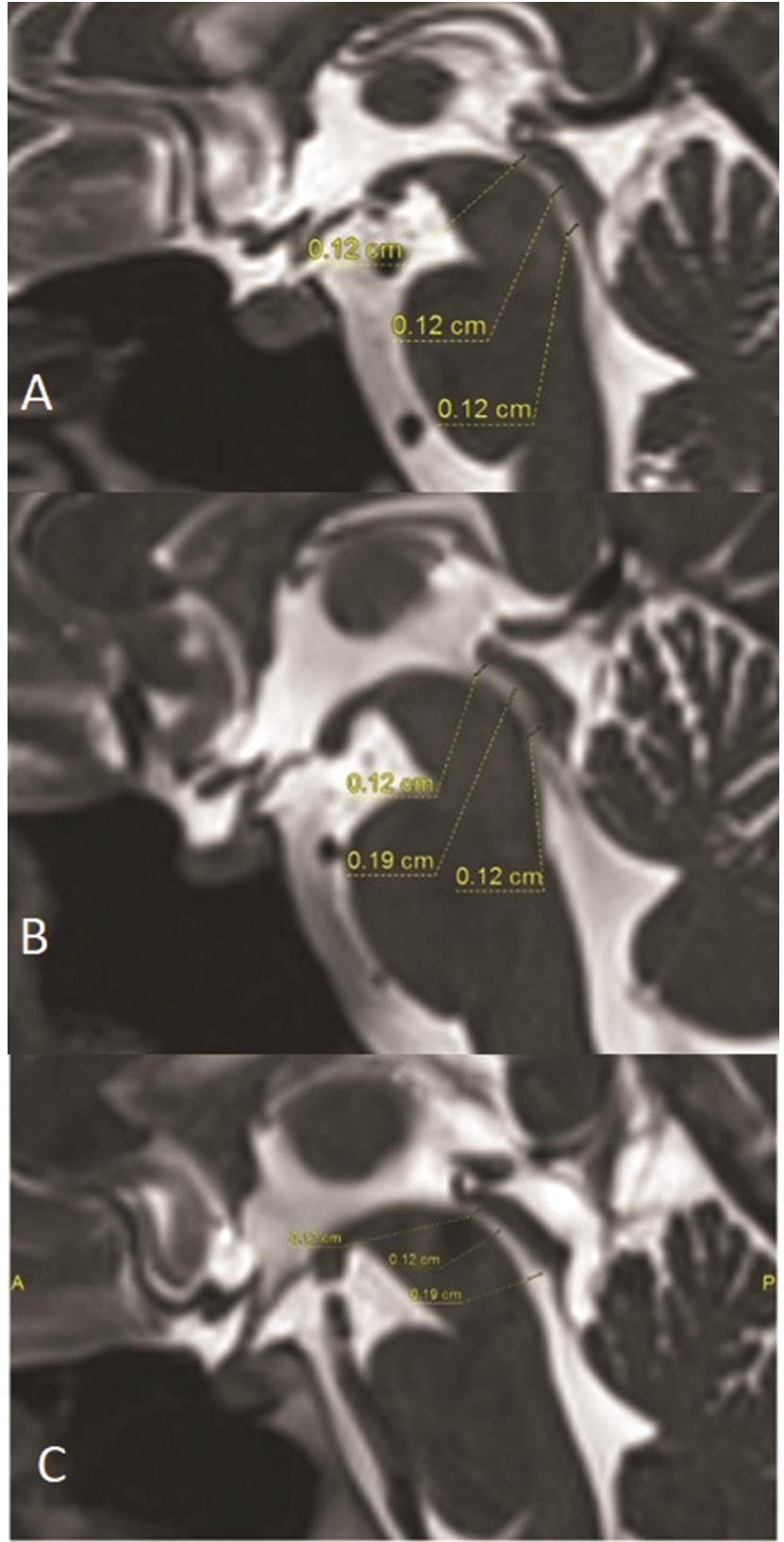

Fig. 2 T2-weighted 3D Sampling Perfection with Application optimized Contrasts using different flip-angle Evolution (SPACE) MRI images of brain in sagittal plane $(\mathbf{A}-\mathbf{C})$ showing the three patterns (I-III) of aqueduct.
Table 2 Prevalence of types of cerebral aqueduct

\begin{tabular}{|l|l|l|l|}
\hline S. no. & Types & Males (\%) & Females (\%) \\
\hline 1 & Type I & 64 & 44 \\
\hline 2 & Type II & 25 & 50 \\
\hline 3 & Type III & 8 & 4 \\
\hline 4 & Type IV & 3 & 2 \\
\hline
\end{tabular}

the most common type was type II (50\%) followed by type I (45\%) and type III (4\%). The type IV was seen in less than $5 \%$ in both males and females. There was no statistically significant difference between males and females in these four types of aqueduct.

The mean angle between AC-PC line and the cranial part of aqueduct was $51^{\circ} \mathrm{in}$ males and $53^{\circ} \mathrm{in}$ females; mean angle between the cranial and caudal part of aqueduct was $28^{\circ}$ in males and $26^{\circ}$ in females; the mean angle between the caudal part of aqueduct and the fourth ventricles was $12^{\circ}$ in both males and females. There was no statistically significant difference in the angles between males and females. The interobserver correlation coefficients for the length and the diameter of the cerebral aqueduct were 0.72 and 0.79 , respectively.

\section{Discussion}

Cerebral aqueduct represents the primitive mesencephalic cavity. Since the course of the aqueduct concave is ventral, the length of the aqueduct was described as cranial and caudal parts. The angulation of its course is taken as point of division of both parts. ${ }^{3}$ The mean value of the length of the cranial and caudal part of aqueduct in males were $6.9 \mathrm{~cm}$ and $8.6 \mathrm{~cm}$ and in females $6.5 \mathrm{~cm}$ and $8.0 \mathrm{~cm}$, respectively. The length was more in males than females with significance ( $p=0.006$ and 0.02 , respectively). Mean total length of aqueduct in our study was more, compared to the study by Matys et al. ${ }^{3}$ However, they measured the overall length without division of cranial and caudal parts. In the present study, the length of the cranial part was more when compared to the caudal part in both males and females. There was significant difference between the lengths of the two parts (cranial part was more). This may be of significance in endoscopic approach of the cranial and caudal ends of the 
aqueduct through foremen of Munro and through posterior medullary velum, respectively. ${ }^{4}$

At the same time, due to the angulation on its course, angles were measured at different levels. The mean angle between tangential line and the cranial part of aqueduct (angle A1) was $51^{\circ}$ in males and $53^{\circ}$ in females; mean angle between the cranial and caudal parts of aqueduct (angle A2) was $28^{\circ}$ in males and $26^{\circ}$ in females; the mean angle between the caudal part of aqueduct and the floor of the fourth ventricle (angle A3) was $12^{\circ}$ in both males and females. There was no statistically significant difference in the angles (angle A1, A2, A3) between males and females. The angle between the cranial and caudal part was more in the study by Matys et al. In the present study, angle A1 was more compared to angle A2 and angle A3. This can be due to racial differences in the shape of the skull. These angles may be important in avoiding injury to the tectal plate during transfrontal aqueductoplasty, because of the trajectory of endoscopic tube through foramen of Munro towards the aditus of aqueduct. ${ }^{3}$ Angle A2 and angle A3 are relevant in approaching the aqueduct through fourth ventricle to avoid injury to the adjacent nuclei and tectal plate. The ocular motility disturbance, due to the inadvertent tectal plate injury during endoscopic procedures, can be minimized with the help of the morphometric measurements. ${ }^{5}$

The diameter of the aqueduct was measured at three levels-at the cranial end of the aqueduct (D1), the middle part (D2), and at the caudal part (D3). The mean diameter of the aqueduct at cranial, middle and caudal part in males were $1.2 \mathrm{~cm}, 1.3 \mathrm{~cm}$ and $1.8 \mathrm{~cm}$, respectively. The mean diameter of aqueduct at cranial, middle, and caudal part in females were $1.2 \mathrm{~cm}, 1.3 \mathrm{~cm}$ and $1.7 \mathrm{~cm}$, respectively. There was no statistically significant difference in D1, D2 and D3 between males and females. This mean value may be of help in choosing the size in endoscopic neurosurgical procedures like aqueductoplasty and stunt replacement. ${ }^{4}$ But the shape of the aqueduct varies from the third to fourth ventricle. ${ }^{5,6}$ In the literature, the morphology has been described as two constrictions with an ampulla in between the constrictions. The constrictions were considered very significant for the ease of the endoscopic advancement during neurosurgical procedure in addition to determining the size. Since it was a narrowest part, minimal collection of blood or debris can lead to blockage of aqueduct which, in turn, leads to hydrocephalus or ventriculomegaly. From the present observations, the morphology does not follow the strict pattern of pars anterior followed by first constriction, ampulla, second constriction and pars posterior. ${ }^{3,5,7}$ We have observed that the ampullary part also present near the caudal end of the aqueduct, and in few MR images, there was no ampullary part.

-Figure 3 shows the schematic representation of the types of cerebral aqueduct. We used the measurements in three patients suspected of aqueductal stenosis and found that the obstruction at proximal, mid-, and distal parts of aqueduct are delineated better. The case of proximal obstruction ( - Fig. 4A) showed normal diameters in the mid- and distal parts of the aqueduct. In midpart and distal aqueductal obstruction, there was mild upstream dilatation of the proximal aqueduct ( $\mathbf{- F i g}$. $\mathbf{4 B}, \mathbf{C}$ ). Further studies comparing these diameter and angle measures in suspected aqueductal pathology would help to establish the role of these measures. Moreover, these types can be an initiative to find alternate and easy approach to the cerebral aqueduct by endoscopy, so that intraoperative complications like basilar artery injury, ${ }^{8}$ tectal plate injury, and subsequent postoperative morbidity are minimized. The detailed clinical significance of

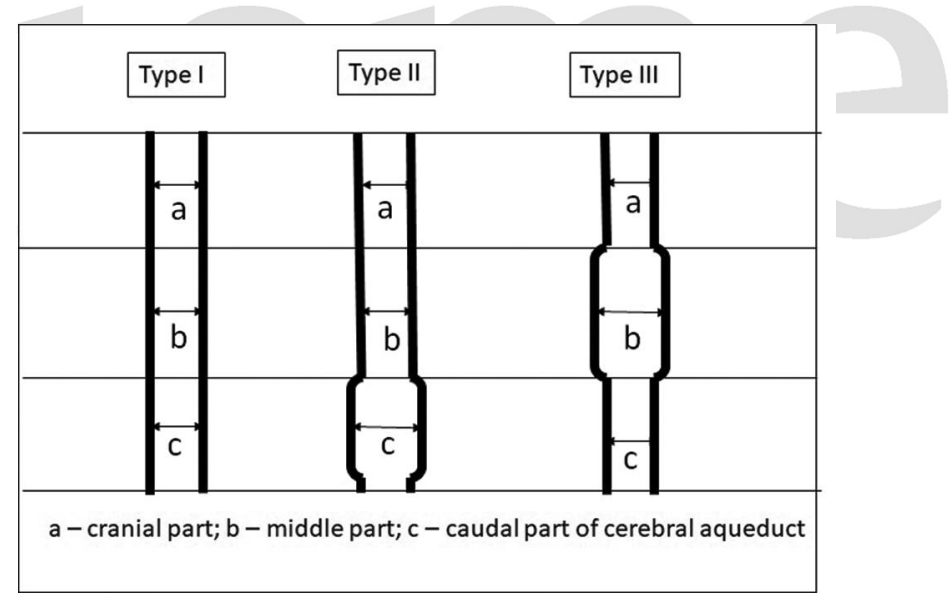

Fig. 3 Schematic representation of three types of cerebral aqueduct.

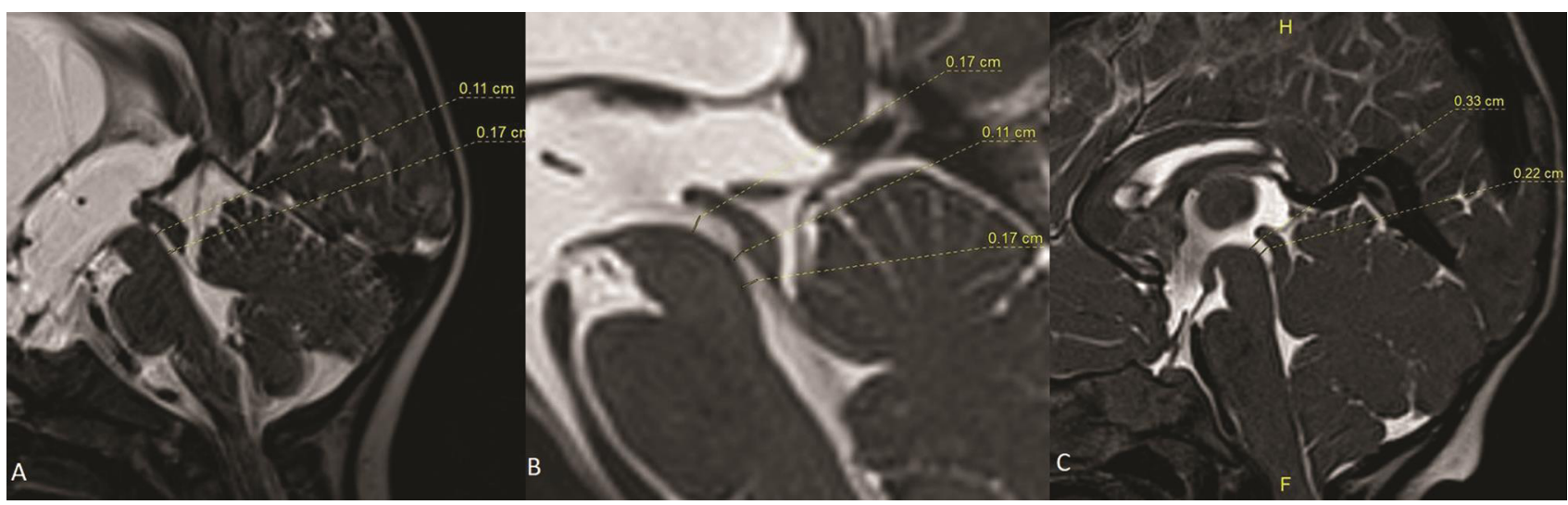

Fig. 4 (A-C) Sagittal T2-weighted 3D Sampling Perfection with Application optimized Contrasts using different flip-angle Evolution (SPACE) MRI images in three different patients with proximal, mid- and distal parts of aqueduct showing the measurements. 
these types and the sensitivity of the types of the aqueduct in neurosurgical and neuroradiological applications have to be evaluated clinically. The precise knowledge about the morphometry of cerebral aqueduct may also provide a reference value in diagnosing the aqueductal stenosis and also perhaps in the management of neurosurgical conditions like blockage, narrowing, or idiopathic stenosis of cerebral aqueduct. ${ }^{2,9}$

The morphometric measurements of aqueduct based on MRI enhances precise knowledge about anatomy of the cerebral aqueduct. It also helps the radiologist to diagnose the pathology involving the aqueduct like stenosis, narrowing, or developmental anomaly which causes increased intracranial pressure. It also provides a basic morphological data to the surgeons that can be helpful in endoscopic neurosurgeries, in order to minimize the intraoperative complications, thereby avoiding the postoperative morbidity and its associated hospital stay.

\section{Conflict of Interest}

None declared.

\section{References}

1 Standring S, Gray's Anatomy: The anatomical basis of clinical practice. 21st ed. New York, Elsevier; 2016

2 da Silva LR, Cavalheiro S, Zymberg ST. Endoscopic aqueductoplasty in the treatment of aqueductal stenosis. Childs Nerv Syst 2007;23(11):1263-1268

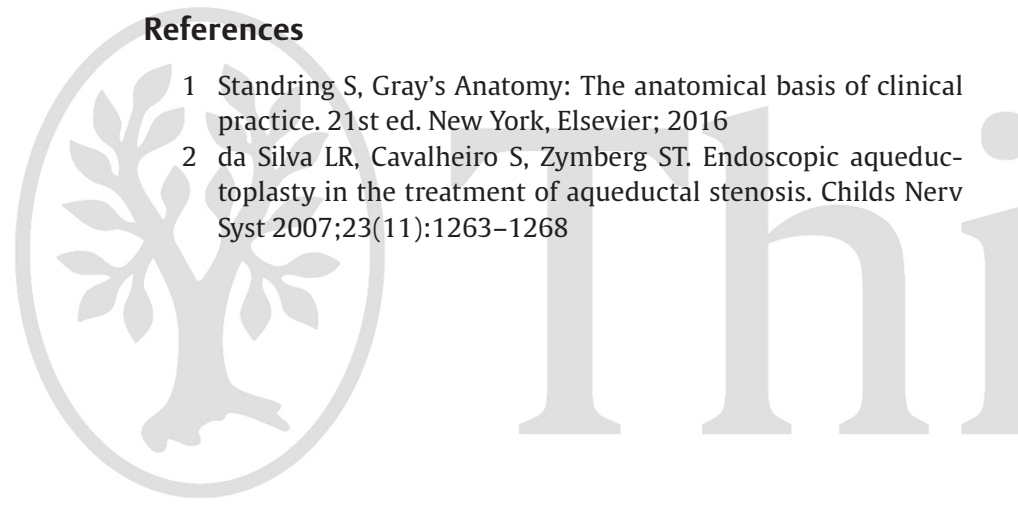

3 Matys T, Horsburgh A, Kirollos RW, Massoud TF. The aqueduct of sylvius: Applied 3-T magnetic resonance imaging anatomy and morphometry with neuroendoscopic relevance. Oper Neurosurg (Hagerstown) 2013;73:ons132-140

4 Duffner F, Schiffbauer H, Glemser D, Skalej M, Freudenstein D. Anatomy of the cerebral ventricular system for endoscopic neurosurgery: a magnetic resonance study. Acta Neurochir (Wien) 2003;145(5):359-368

5 Longatti P, Fiorindi A, Perin A, Martinuzzi A. Endoscopic anatomy of the cerebral aqueduct. Neurosurgery 2007;61(3, Suppl):1-5

6 Aziz A, Hu Q Nowinski WL. Morphometric analysis of cerebral ventricular system from MR images. Medical Imaging: Physiology, Function, and Structure from Medical Images 2004;5369:574-583

7 Woollam DH, Millen JW. Anatomical considerations in the pathology of stenosis of the cerebral aqueduct. Brain 1953;76(1):104-112

8 Schroeder HW, Oertel J, Gaab MR. Endoscopic aqueductoplasty in the treatment of aqueductal stenosis. Childs Nerv Syst 2004;20(11-12):821-827

9 Akyer SP, Cagirici S, Ozdemir MB. Relationship of age with the size of the interventricular foramina and aqueductus sylvii: a morphometric evaluation. Neurol Res 2014;36(10):878-881
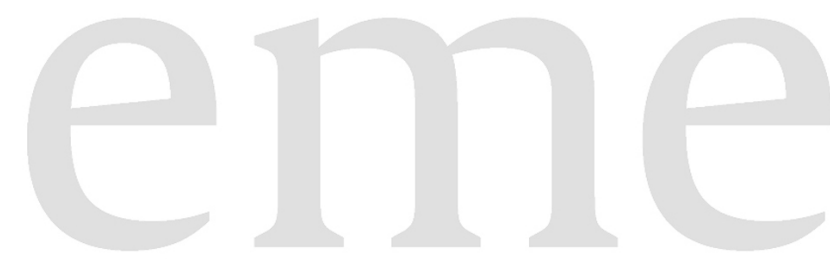